\title{
Proportional myoelectric control of a virtual object to investigate human efferent control
}

Received: 5 December 2003 / Accepted: 26 March 2004 / Published online: 16 July 2004

(C) Springer-Verlag 2004

\begin{abstract}
We used proportional myoelectric control of a one-dimensional virtual object to investigate differences in efferent control between the proximal and distal muscles of the upper limbs. Eleven subjects placed one of their upper limbs in a brace that restricted movement while we recorded electromyography (EMG) signals from elbow flexors/extensors or wrist flexors/extensors during isometric contractions. By activating their muscles, subjects applied virtual forces to a virtual object using a real-time computer interface. The magnitudes of these forces were proportional to EMG amplitudes. Subjects used this proportional EMG control to move the virtual object through two tracking tasks, one with a static target and one with a moving target (i.e., a sine wave). We hypothesized that subjects would have better control over the virtual object using their distal muscles rather than using their proximal muscles because humans typically use more distal joints to perform fine motor tasks. The results indicated that there was no difference in subjects' ability to control virtual object movements when using either upper arm muscles or forearm muscles. These results suggest that differences in control accuracy between elbow joint movements and wrist joint movements are more likely to be a result of motor practice, proprioceptive feedback or joint mechanics rather than inherent differences in efferent control.
\end{abstract}

Keywords Motor learning - Motor control · Electromyography $\cdot$ Human-computer interface

K. E. Gordon $(\bowtie) \cdot$ D. P. Ferris Department of Movement Science, University of Michigan, 401 Washtenaw Avenue, Ann Arbor, MI 48103-2214, USA

e-mail: kegordon@umich.edu

Tel.: +1-734-6151711

Fax: +1-734-9361925

\section{Introduction}

People routinely choose to use certain limb segments to perform particular types of movements. For example, people normally use their hands and fingers to perform fine motor tasks such as writing, drawing, and typing. In contrast, people primarily use proximal upper limb segments for gross motor tasks such as lifting, throwing, and swimming. This distal to proximal trend for fine to gross motor movements largely results from the mechanics of human anatomy. It is easier to thread a needle with small movements of your fingers than it is to rotate your whole upper limb about the shoulder. However, it is not clear if this difference in normal motor behavior reflects limitations of motor control between the distal and proximal muscles of the upper limb.

Three factors could potentially lead to differences in motor performance between distal and proximal joints:

1. Afferent feedback

2. Joint mechanical properties

3. Efferent control

Techniques have been developed to study these factors. Potential differences in afferent feedback can be identified by examining densities and sensitivities of muscle spindles (Goodwin et al. 1972; Hall and McCloskey 1983; De Domenico and McCloskey 1987; Jones et al. 2001), Golgi tendon organs (Lundberg et al. 1978; Gregory and Proske 1979; Prochazka and Wand 1980; Crago et al. 1982; Jami 1992), joint proprioceptors (Macefield et al. 1990; Gandevia et al. 1992), and cutaneous receptors (Moberg 1983; Rao and Gordon 2001). Joint mechanical properties have been well quantified using system identification methods (Hogan 1985; Mussa-Ivaldi et al. 1985; Gottlieb 1996; Stein et al. 1996; Kearney et al. 1997; Kirsch and Stein 1997; Mirbagheri et al. 2000). Lastly, efferent control is possibly the most difficult of the three factors to study because it is confounded by the other two factors. To study efferent control with limited effects from afferent feedback and joint mechanical properties, neuroscientists rely on techniques such as direct cortical recordings (Taira 
et al. 1996; Georgopoulos et al. 1999; Moran and Schwartz 1999; Schwartz and Moran 2000; Reina et al. 2001) and functional magnetic resonance imaging or positron emission tomography (Dettmers et al. 1996a, 1996b; Thickbroom et al. 1998; Nirkko et al. 2001). However, these techniques record brain representations of efferent control and bypass spinal effects on efferent commands (Bizzi et al. 2000).

The purpose of this study was to quantify differences in efferent control between proximal and distal muscles of the upper limb using a novel method. Subjects completed a series of tracking tasks by moving a virtual object on a video screen. Subjects controlled movements of the virtual object using proportional electromyography (EMG) signals from distal or proximal muscles of their upper limb. An arm restraint prevented movement of the upper limb so that subjects used isometric muscle activation to modify EMG amplitudes. This setup allowed us to examine differences in efferent control between muscle groups independent of variations in joint mechanical properties. In addition, this procedure may decrease potential differences in proprioceptive feedback in controlling the virtual object movements during the task.

Based on behavior in everyday tasks, we hypothesized that subjects would exhibit greater control over the virtual object using EMG signals from more distal muscles of the upper limb than when using more proximal muscles. We based this hypothesis on the normal uses of proximal and distal muscles during normal motor behavior. Humans tend to use their distal joints for fine motor tasks and their proximal joints for more gross motor tasks.

\section{Materials and methods}

\section{Subjects}

Eleven subjects ( 7 female and 4 male) participated in this study (age: $24 \pm 4.6$ years, mean \pm SD). Subjects were healthy and classified themselves as right arm dominant. All subjects provided informed written consent. The University of Michigan Human Subjects Protection Office approved the protocol. All procedures were in accordance with the Declaration of Helsinki.

\section{Testing position}

For all trials, subjects sat upright and placed their right upper limb in a stationary brace, restricting movement at the shoulder, elbow, and wrist joints (Fig. 1). We adjusted the height of the brace so that each subject's upper limb was parallel to the floor. The subject's shoulder was horizontally flexed at approximately $20^{\circ}$. The elbow was flexed at approximately $45^{\circ}$ from full extension. The wrist was held in the neutral position. An oscilloscope in front of the subject provided visual feedback about the position of the virtual object and tracking target.

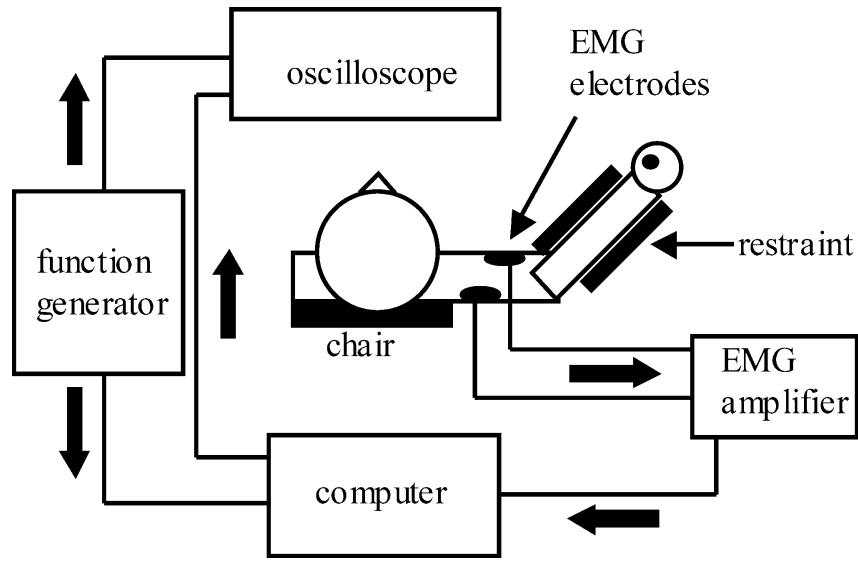

Fig. 1 Subjects sat with their right arm placed in a restraining device that restricted motion at the shoulder, elbow, and wrist joints. A computer processed EMG data from either forearm flexors/ extensors or biceps/triceps muscles to control one-dimensional movement of a virtual object. An oscilloscope displayed the position of the virtual object in front of the subject. A function generator created a tracking target. The target was displayed on the oscilloscope and recorded by the computer

\section{Electromyography}

We collected EMG data from four muscle groups of the right upper limb. We placed rectangular bipolar silversilver chloride surface electrodes $(1.0$ by $0.5 \mathrm{~cm}$, center-tocenter distance of $2.5 \mathrm{~cm}$ ) over the following muscle groups (Basmajian and Blumenstein 1989):

1. Biceps brachii

2. Medial head of the triceps brachii

3. Flexor carpi radialis and flexor digitorum superficialis (forearm flexors)

\section{Extensor digitorum}

Electromyography signals were amplified with a signalconditioning amplifier (Konigsberg Instruments Inc., Pasadena, CA, USA) that had a $10-1,000 \mathrm{~Hz}$ bandwidth and sent to a personal computer via an analog to digital board (dSPACE Inc., Northville, MI, USA).

\section{Virtual object interface}

We used a real-time computer interface (dSPACE Inc., Northville, MI, USA) to control a virtual object on an oscilloscope screen. Computer software sampled the EMG signals at $1,000 \mathrm{~Hz}$, high-pass filtered the signals with a second order Butterworth filter $\left(f_{c}=20 \mathrm{~Hz}\right)$, rectified the signals, low-pass filtered the signals with a second order Butterworth filter $\left(f_{c}=10 \mathrm{~Hz}\right)$, zeroed the EMG signal that was less than the level of background noise and normalized the signal to an EMG amplitude recorded during a maximum voluntary isometric contraction. The computer then used the processed signals to generate virtual forces to move a virtual object in one dimension. The forces acting on the virtual object were proportional to the amplitudes of the processed EMG signals. Flexor EMG signals (biceps brachii or forearm flexors) provided forces 
Fig. 2a-d Each graph displays the target position and the virtual object position during the initial and final trial for subject 4. a Static trials using forearm muscle groups to control the virtual object. b Dynamic trials using forearm muscle groups. c Static trial using upper arm muscle groups. d Dynamic trials using upper arm muscle groups. For all static trials the goal of the subject was to move the virtual object from position 0 to the target at position 3 and hold it there for the remainder of the trial. For all dynamic trials the goal of the subject was to move the virtual object from position 0 to the target and to trace the sinusoidal oscillating target for the remainder of the trial
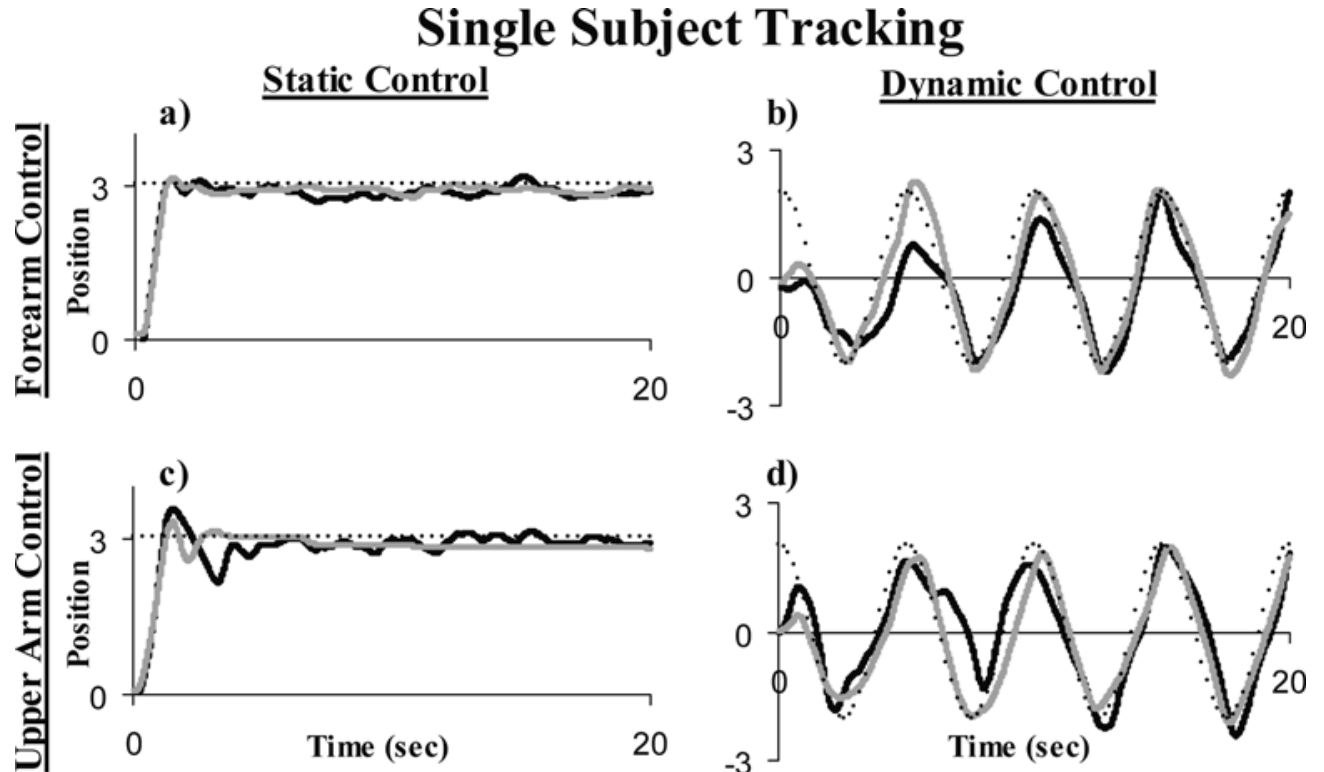

in one direction and extensor EMG signals (triceps brachii or extensor digitorum) provided forces in the opposite direction. The equation governing virtual object movement was:

$\ddot{x}=\frac{(f l e x E M G-\operatorname{ext} E M G)-(c \dot{x})}{m}$

where flexEMG was the high-pass filtered, rectified, and low-pass filtered, normalized EMG signal from the forearm flexors or biceps brachii, extEMG was the highpass filtered, rectified, low-pass filtered, normalized EMG signal from the extensor digitorum or triceps brachii, $m$ was the virtual object mass, $c$ was a damping coefficient reflecting dynamic friction on the virtual object, and $x$ was the one-dimensional position of the virtual object. Thus, the movement of the virtual object was a result of antagonistic forces acting on a system possessing inertial and damping characteristics.

We chose mass ( 0.5 unit-less) and damping coefficient ( 0.5 unit-less) values such that subjects could perform the tracking tasks while keeping EMG amplitudes lower than $25 \%$ of their maximum voluntary isometric contraction EMG amplitude. This allowed the subjects to complete the tasks without experiencing substantial fatigue. This is important because EMG signal characteristics can be affected by fatigue (Viitasalo and Komi 1977). Fatiguerelated changes could potentially increase the difficulty of controlling the virtual object and would complicate the results of this study. The threshold of the EMG processing coupled with the low damping coefficient and mass resulted in the virtual object being accelerated in response to bursts of EMG greater than $5 \%$ of the maximum voluntary isometric contraction (MVC). An effect of the low damping coefficient was that it was difficult to bring the virtual object to rest. Subjects could move the virtual object to the target, but it was difficult to achieve a zero velocity at any given point. The damping was not high enough to quickly bring the object to rest. Subjects typically moved the virtual object on or near the target and then counteracted drift with small bursts of muscle activity.

\section{Tracking tasks}

Subjects isometrically activated their flexor and extensor muscles to position the virtual object over a target. In half the trials, the target was stationary (static task). During the static task subjects had to move the virtual object from a baseline position of zero to a target dimensionless position of 3, and then attempt to hold the virtual object motionless. In the other half of the trials, the target moved in a sine wave pattern (dynamic task) at $0.2 \mathrm{~Hz}$ with dimensionless amplitude of 2.5. During the dynamic task the subjects had to move the virtual object continuously in a sinusoidal fashion. The frequency and amplitude of the sine wave pattern were selected based on pilot studies. At the chosen conditions, movement of the virtual object was found to be initially challenging to subjects but not so challenging that subjects became frustrated. When using frequencies of target movement faster than $0.2 \mathrm{~Hz}$ we found some subjects would give up midway through a trial if they were unable to keep up or catch up to the target. When using frequencies slower than $0.2 \mathrm{~Hz}$ the task became too easy to allow us to make clear discriminations between control ability. The target was produced using a function generator. The target position and virtual object position were overlaid in real time on an oscilloscope. 
Fig. 3a-d Each graph displays the target position during a static 20 -s trial, and the mean and standard deviation of the position of the virtual object calculated from all 11 subjects. For all static trials the goal of the subject was to move the virtual object from position 0 to the target at position 3 and hold it there for the remainder of the trial. a The initial static trial using the forearm muscle groups to control the virtual object. b The final static trial using the forearm muscle groups. c The initial static trial using the upper arm muscle groups. $\mathbf{d}$ The final static trial using the upper arm muscle groups

\section{Group Static Tracking}
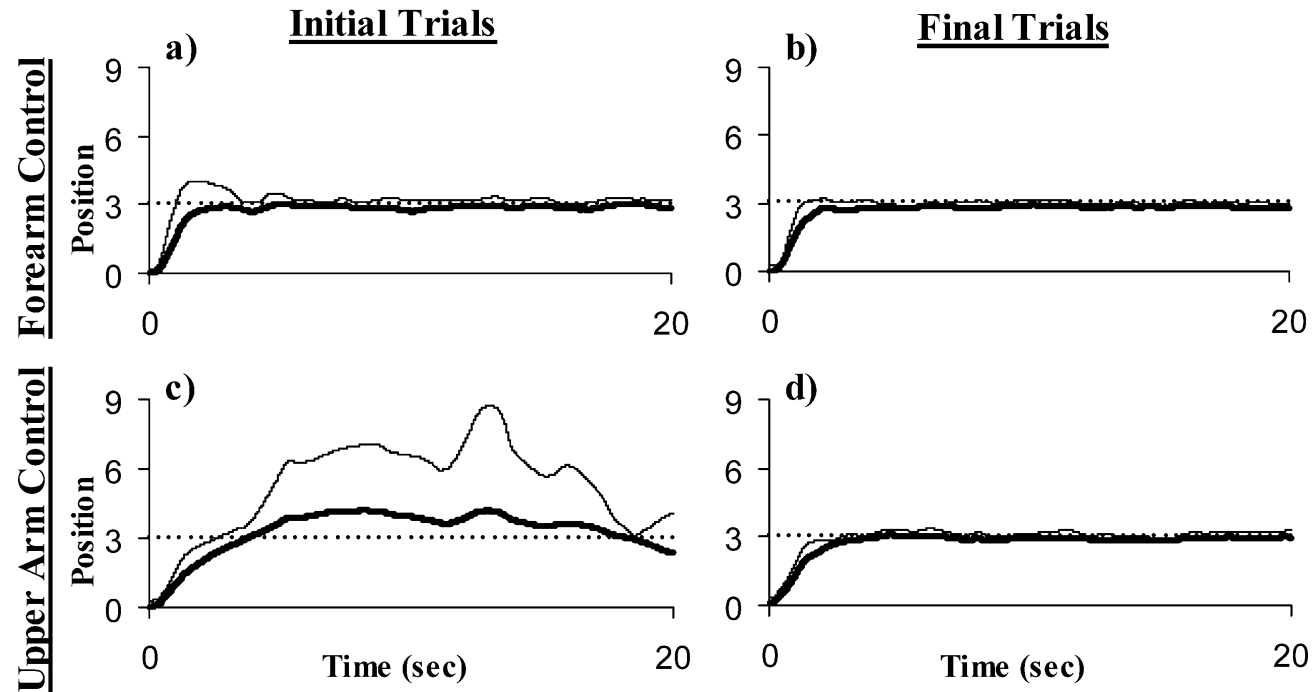

Protocol

Subjects performed the experimental design twice, once using the distal muscles (forearm flexors and extensor digitorum) as controllers and once using the proximal muscles (biceps brachii and triceps brachii) as controllers. The order of the two muscle pairs was randomized across subjects. After placing their right arm in the restraint, subjects performed three trials of a 5-s MVC for each of the muscle groups. We high-pass filtered, rectified, and low-pass filtered the EMG data as described previously. Next, we searched for the greatest average amplitude encompassed by a continuous 1 -s period in each MVC trial. We used the highest average amplitude for each muscle group to normalize all further EMG signals from that muscle group. We used the greatest average EMG amplitude over 1-s of data rather than the entire 5-s to ensure that we captured only the true MVC. This was necessary because the amplitude fluctuated over the 5-s period. For example, EMG amplitude typically increased over the first second of data when subjects went from rest to maximum activation. Next, we recorded three 5-s trials in which subjects were instructed to completely relax their muscles. These EMG data were processed identically to those for the MVC. The average amplitude of relaxed trials was used to determine the baseline noise levels. Subjects then performed 20-s trials of static and dynamic tracking tasks, alternating between the two tasks in that order. Subjects completed ten trials of both static and dynamic tasks. All subjects rested for a minimum of $10 \mathrm{~s}$ between trials. We encouraged subjects to rest for longer periods of time if they felt fatigued, but most subjects declined to take longer rest periods.
Analysis

We analyzed the first and tenth trials of the static and dynamic tracking tasks for both the distal and proximal muscle groups. For each trial, we calculated the root mean square error (RMSE) between the target and the actual position of the virtual object. For the dynamic trials we included all 20-s periods of each trial to calculate RMSE. For the static trials, we included the time beginning when the object first crossed the target position until the end of the trial. If the subject did not move the virtual object across the target position, we began calculating RMSE at the time when progression toward the target first switched from positive to negative (this was only necessary during one trial for one subject). We used a repeated measures ANOVA to test for significant differences in RMSE between trial number (first and tenth), control group (forearm and upper arm), and task (static and dynamic). We set the significance level at $P<0.05$ and used Tukey Honestly Significant Difference Post-Hoc tests where appropriate.

We calculated root mean square (RMS) values on the normalized filtered EMG over the full 20-s trials. We ran two four-way ANOVAs to check for differences in RMS EMG values between trial number, muscle, and task. We set the significance level at $P<0.05$. In addition, for each trial, we calculated the median power frequency between 10 and $500 \mathrm{~Hz}$ for EMG data that were band-pass filtered with a second order Butterworth filter $\left(f_{c}=10-500 \mathrm{~Hz}\right)$. A decrease in median power frequency of the EMG signal with muscle has been shown to be associated with fatigue (Ng et al. 1996). We used a four-way ANOVA to test for differences in median power frequency between trial number, muscle, test condition, and subject. We set the significance level at $P<0.05$ and used Tukey Honestly Significant Difference Post-Hoc tests where appropriate. 


\section{Results}

During the initial trials, subjects appeared to have slightly better control of the virtual object with their forearm muscles than with their upper arm muscles (Figs. 2, 3, 4), but this difference was not significant. Although the means and standard deviations of the RMSE were larger for upper arm control than for forearm control (Table 1), there were no statistical differences for control group $\left(F_{(1,74)}=2.4694, P=0.1204\right)$. This could be partly attributed to the large inter-subject variation in upper arm control. However, ANOVA results reveal an effect size of only 0.16 for muscle group. Thus, if there was a real difference in control between the upper arm and forearm muscles (Type II Error), the difference was small.

The control accuracy between the two control groups was practically identical after the ten trials performed in the experiment. Subjects substantially improved virtual object control with practice (Figs. 2, 3, 4) and the improvements were greater for upper arm control than for forearm control (Table 1). The improvements with practice resulted in a significant main effect for trial number $\left(F_{(1,74)}=7.5271, P=0.0076\right)$. There were no significant differences for task $\left(F_{(1,74)}=1.6635, P=0.2011\right)$.

Although the subjects improved their performance in both tasks, there were no obvious changes in muscle activation patterns for the dynamic task (Figs. 5, 6). There appears to be a trend that subjects decreased levels of muscle coactivation during the static task (Figs. 7, 8) but statistical analysis revealed no differences. There were no significant differences in RMS EMG values between the initial and final trials for any muscle group during performance in either the static $\left(F_{(5,82)}=0.8251\right.$, $P=0.5354)$ or dynamic task $\left(F_{(5,80)}=1.6699, P=0.1514\right)$.
There were no significant differences in median power frequency with trial number $\left(F_{(1,160)}=1.2084, P=0.2733\right)$, suggesting that subjects did not become fatigued during the experiment. There were significant differences in median power frequency between static and dynamic tasks $\left(F_{(1,160)}=33.3602, \quad P<0.0001\right)$ and between muscles $\left(F_{(3,160)}=10.4585, P<0.0001\right)$. Post hoc tests revealed biceps' median power frequency was different from that of the triceps and forearm extensors. Forearm extensor median power frequency was different from forearm flexors.

\section{Discussion}

Based on these data forearm efferent control is not more accurate than upper arm efferent control. The initial mean trajectory of the virtual object demonstrated differences between forearm and upper arm control (Figs. 2, 3), but the differences were not significant and variability between subjects was high. With just ten trials of practice, subjects were able to greatly increase virtual object control with their upper arm muscles to match the control accuracy of their forearm muscles. This finding suggests that differences in control accuracy between the elbow joint and the wrist joint are more likely to be a result of joint mechanics, proprioceptive feedback, or motor practice, rather than inherent differences in efferent control. Practice in the form of life experience might explain the initial differences we observed between forearm and upper arm control. It is possible that everyday tasks for the forearm more closely resemble the tasks in this experiment than everyday upper arm tasks. This everyday usage may have given subjects an initial advantage using forearm control. However, these differences did not last once the
Fig. 4a-d Each graph displays the target position during a dynamic 20 -s trial, and the mean and standard deviation of the position of the virtual object calculated from all 11 subjects. For all dynamic trials the goal of the subject was to move the virtual object from position 0 to the target and to trace the sinusoidal oscillating target for the remainder of the trial. a The initial dynamic trial using the forearm muscle groups to control the virtual object. b The final dynamic trial using the forearm muscle groups. c The initial dynamic trial using the upper arm muscle groups. d The final dynamic trial using the upper arm muscle groups

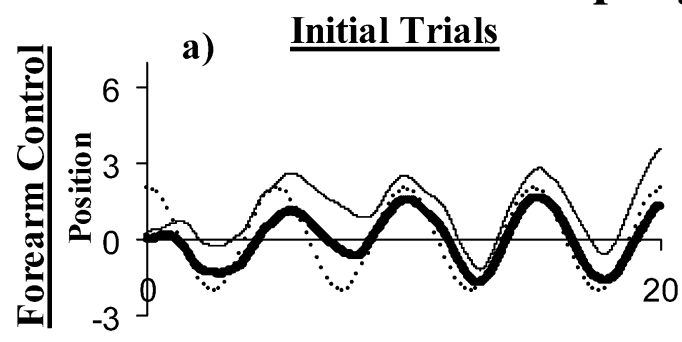

Group Dynamic Tracking

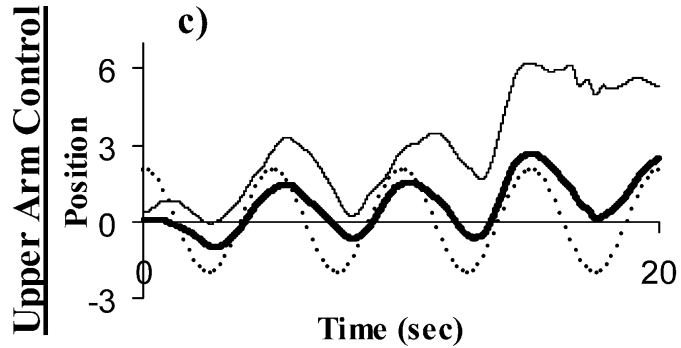

b) Final Trials

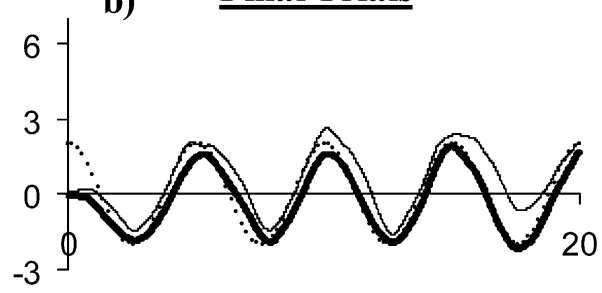

d)

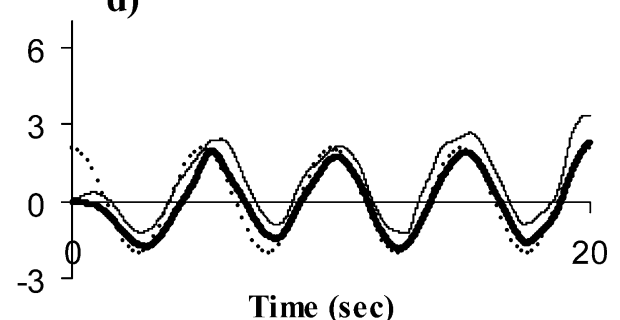

Time (sec)

$\bullet \bullet \bullet$ Target $\longrightarrow$ Mean —SD 
Table 1 Root mean square error (mean \pm SD) of the virtual object position (dimensionless) relative to the target position for the four tracking tasks. The only significant difference found was between the initial and final trials $(P<0.05)$

\begin{tabular}{lcccc}
\hline & Static (initial) & Static (final) & Dynamic (initial) & Dynamic (final) \\
\hline Forearm & $0.79 \pm 0.21$ & $0.68 \pm 0.09$ & $1.18+0.63$ & $0.85+0.45$ \\
Upper arm & $1.48 \pm 1.96$ & $0.66 \pm 0.09$ & $1.81 \pm 2.13$ & $0.82 \pm 0.28$ \\
\hline
\end{tabular}

subjects were given practice performing the task with the upper arm.

The findings of this study are in agreement with research demonstrating that the ability to control force accuracy is no different between distal and proximal muscle groups of the upper limb (Gandevia and Kilbreath 1990; Jones 2000). In these past studies, subjects estimated the level of force being produced or maintained specific levels of force to match a static target. In our study, subjects were able to control the efferent output of their forearm muscles and upper arm muscles equally well after a very small amount of practice (ten trials). An important difference between our study and the prior experiments is that our study removed the potential effects of muscle-tendon geometry and joint mechanics. Subjects were only required to modulate muscle activation for control rather than the mechanical output of force. In addition, the present study also expands on the prior research because our subjects performed dynamic modulations of efferent output as well as static modulations.

One limitation of the study is that proprioceptive feedback was not entirely removed from the experiment. Subjects had access to information from cutaneous sensors, Golgi tendon organs, and muscle spindles in their upper limb as they produced isometric contractions against the arm restraint. The role of proprioceptive feedback was limited in this study because it did not provide direct feedback about the position of the virtual object. Visual feedback was the only direct feedback subjects received about the dynamics of the virtual object. Future experiments could study subjects undergoing temporary nerve block from ischemia or anesthetic agent to truly isolate efferent control independent of proprioceptive feedback.

Another possible limitation of this study is the specific method used to process the EMG data. Converting EMG to the position of a virtual object may mask differences in efferent control. If EMG signals have similar spectral content, the variance of linear envelope about its mean would depend mostly on the processing. However, the median power frequency results (Table 2) indicate there were significant differences in spectral content between muscle groups. Although there were no significant differences in median power frequency between the initial and final trials, there were trends for higher median power frequencies with practice. These results suggest that the EMG processing method was not a limiting factor in this study. It would be interesting to repeat a similar protocol
Fig. 5a, b Mean rectified and low-pass filtered EMG from subject 4 over the entire 20 -s period for the dynamic tasks. Forearm extensor and triceps signals were multiplied by -1 for display purposes. Target data are displayed to indicate the subject's temporal goal

\section{Single Subject EMG - Dynamic Trials}
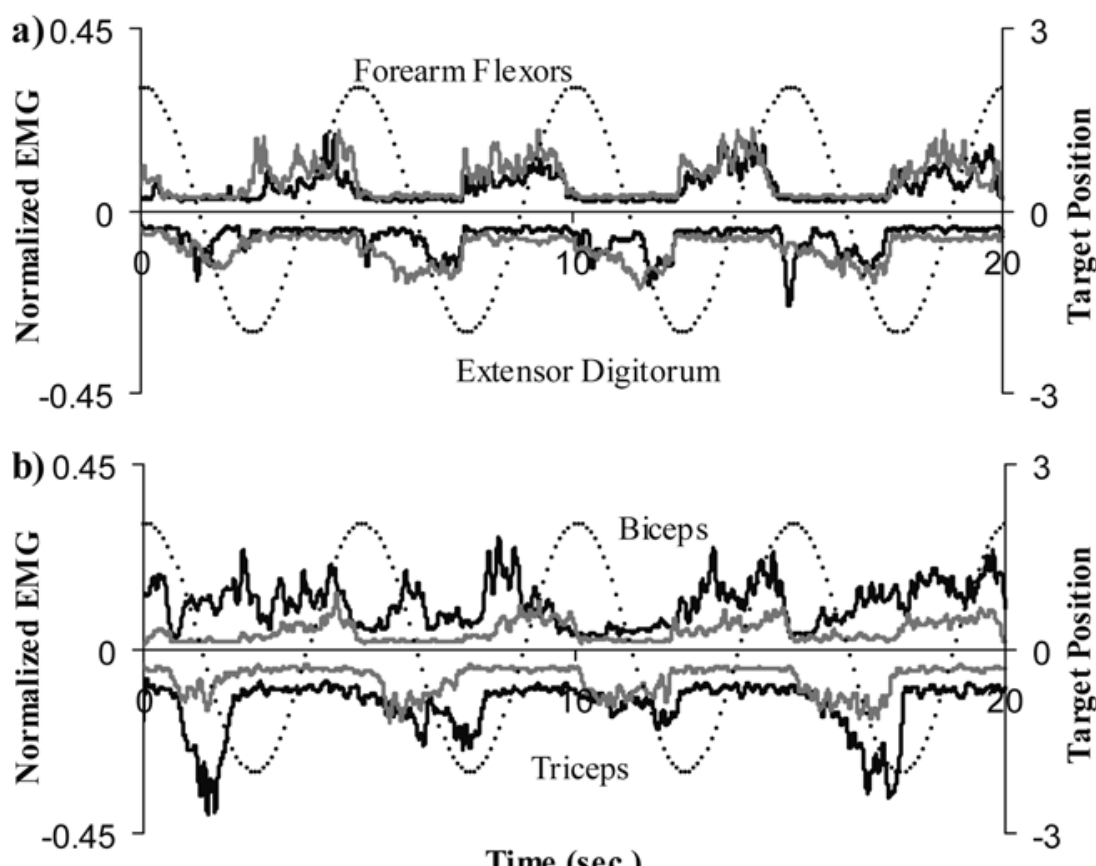

Time (sec.) 
Fig. 6a, b Mean rectified and low-pass filtered EMG from the 11 subjects over the entire $20 \mathrm{~s}$ period for the dynamic tasks. Forearm extensor and triceps signals were multiplied by -1 for display purposes. Target data are displayed to indicate the subjects' temporal goal

\section{Group EMG - Dynamic Trials}
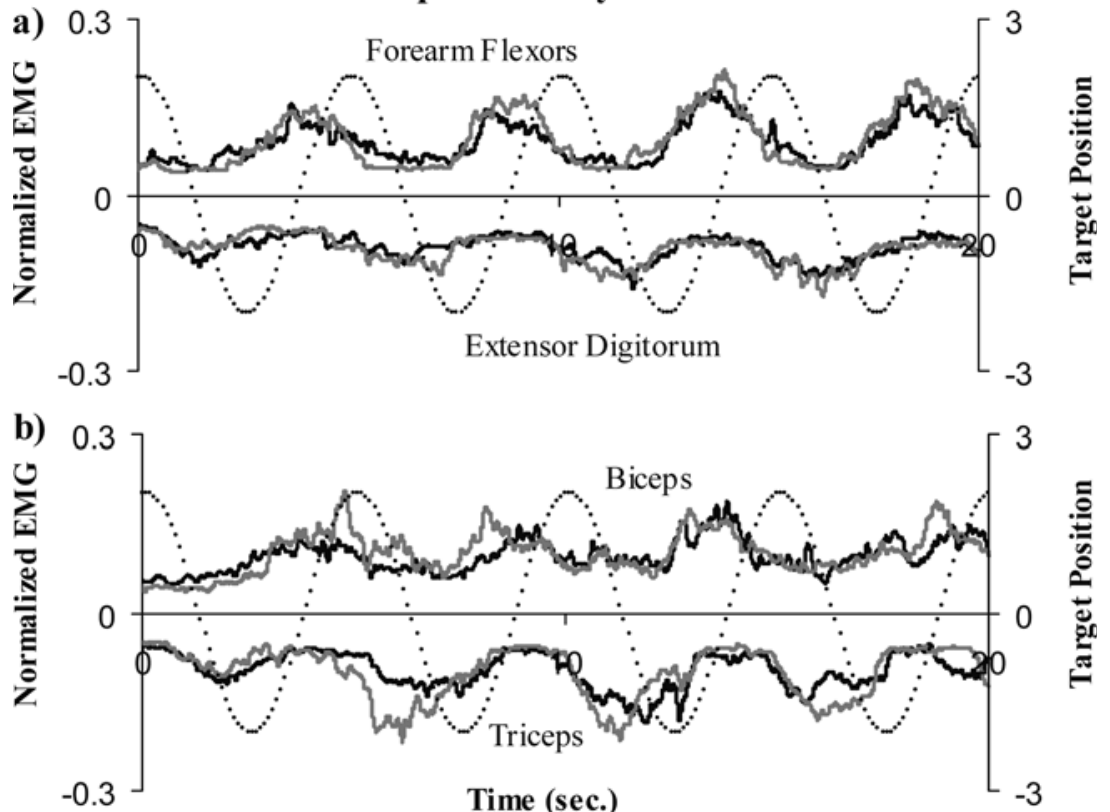

Time (sec.)

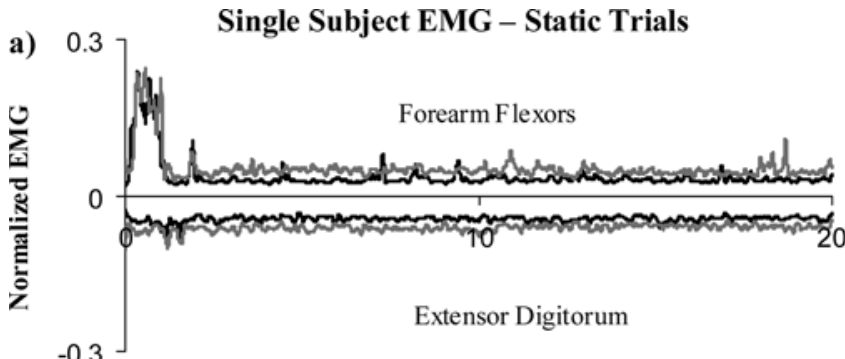

b)

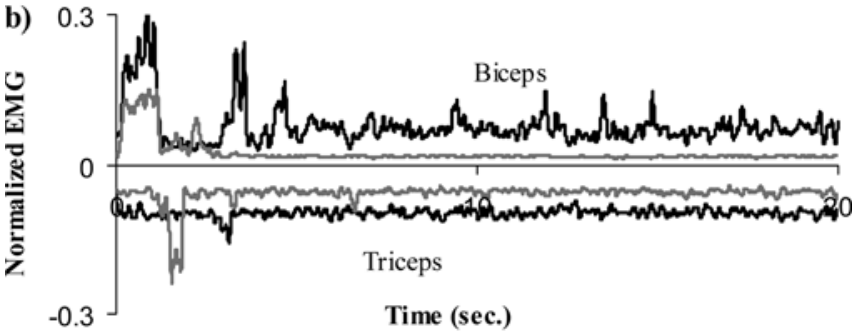

EMG Initial — EMG Final

Fig. 7a, b Mean, rectified and low-pass filtered EMG from subject 4 over the entire 20 -s period during the static positioning task. Forearm extensor and triceps signals were multiplied by -1 for display purposes. Target data are not displayed because the target is constant

using single motor unit EMG to remove most of the signal processing effects.

Due to the nature of the tracking tasks in this study, it was not possible to compare the variance of the linear envelope with its mean for the different muscles. In the static task, subjects produced one main burst of muscle activity at the beginning of the trial and then produced small, scattered bursts to correct virtual object drift. In the
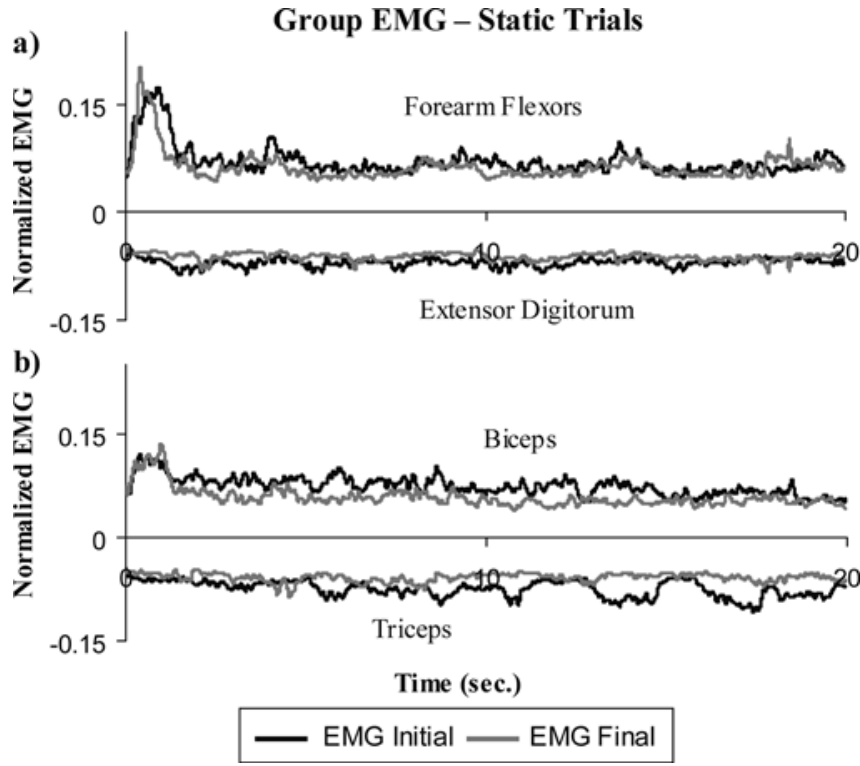

Fig. 8a, b Mean, rectified and low-pass filtered EMG from the 11 subjects over the entire 20 -s period during the static positioning task. Forearm extensor and triceps signals were multiplied by -1 for display purposes. Target data are not displayed because the target is constant

dynamic task, subjects continually modulated muscle activity amplitude. It would be necessary to have subjects produce a constant low amplitude muscle activation (e.g., $15 \% \mathrm{MVC}$ ) to compare the mean and variance in this manner. Future studies could incorporate this procedure to document differences in EMG envelope variance.

An interesting use of this testing protocol would be to examine myoelectric efferent control of virtual objects comparing upper limb muscles and lower limb muscles. If 
Table 2 Electromyography median power frequency (mean and standard deviation) from all 11 subjects during the 20 -s trials. No significant differences were found between initial and final trials $(P>0.05)$. Significant differences were found between static and dynamic tasks $(P<0.05)$ and between muscle groups $(P<0.05)$. Biceps were different from triceps and forearm extensors. Forearm extensors were different from forearm flexors

\begin{tabular}{lrrrrr}
\hline & Static & \multicolumn{3}{c}{ Dynamic } \\
\cline { 2 - 3 } \cline { 5 - 6 } & Initial & Final & Initial & Final \\
\hline Forearm flexors & 115 & 131 & 93 & 98 \\
Mean & 41 & 57 & 21 & 23 \\
Standard deviation & 150 & 150 & 133 & 124 \\
Forearm extensors & 70 & 62 & 45 & 32 \\
Mean & & & & \\
Standard deviation & 99 & 130 & 65 & 85 \\
Biceps & 50 & 76 & 13 & 63 \\
Mean & & & & & \\
Standard deviation & 162 & 160 & 106 & 100 \\
Triceps & 69 & 65 & 28 & 23 \\
Mean & & & & & \\
Standard deviation & & &
\end{tabular}

there are inherent differences in efferent control between any muscle groups, it would seem likely that lower limb muscles might be the least accurate. Humans rarely perform fine motor tasks with their lower limbs, instead relying on them for gross power output during locomotion.

A practical application of myoelectrically controlled virtual objects concerns motor adaptation to powered prostheses. Engineers have dedicated an enormous amount of effort to designing signal processing algorithms for the myoelectric control of upper limb prostheses (Scott 1966; Hogan 1976; Evans et al. 1984; Scott and Parker 1988; Sears and Shaperman 1991; Hudgins et al. 1993; Gallant et al. 1998; Zecca et al. 2002). Yet, there has been much less effort dedicated to understanding the human side of the human-machine interaction in powered prosthesis use. A few studies have examined human motor adaptation to myoelectric prostheses with static control (Gaines 1969; Scott et al. 1978; Paciga et al. 1980; Lovely et al. 1990; Morin et al. 1993; Dupont and Morin 1994), but we could find no studies examining human motor adaptation with proportional control. This is remarkable given that training is one of the most important factors affecting acceptance of the prosthesis (Millstein et al. 1986; Roeschlein and Domholdt 1989; Gaine et al. 1997). While most commercially available myoelectric prostheses use static control, prosthesis engineers have long advocated proportional control to increase function (Hogan 1976). One step toward achieving a useful proportional myoelectric prosthesis would be to study how humans learn to control virtual objects with proportional myoelectric signals. Studying myoelectric control of virtual objects can even provide additional insight into the basic processes of motor control and learning (Manal et al. 2002).
Acknowledgements The authors would like to thank the members of the University of Michigan Human Neuromechanics Laboratory for their insights and contributions to this project. Supported by NIH R01NS045486.

\section{References}

Basmajian JV, Blumenstein R (1989) Electrode placement in electromyographic biofeedback. In: Basmajian JV (ed) Biofeedback: principles and practice for clinicians. Williams and Wilkins, Baltimore, pp 369-382

Bizzi E, Tresch MC, Saltiel P, d'Avella A (2000) New perspectives on spinal motor systems. Nat Rev Neurosci 1:101-108

Crago PE, Houk JC, Rymer WZ (1982) Sampling of total muscle force by tendon organs. J Neurophysiol 47:1069-1083

De Domenico G, McCloskey DI (1987) Accuracy of voluntary movements at the thumb and elbow joints. Exp Brain Res $65: 471-478$

Dettmers C, Connelly A, Stephan KM, Turner R, Friston KJ, Frackowiak RS, Gadian DG (1996a) Quantitative comparison of functional magnetic resonance imaging with positron emission tomography using a force-related paradigm. Neuroimage 4:201-209

Dettmers C, Lemon RN, Stephan KM, Fink GR, Frackowiak RS (1996b) Cerebral activation during the exertion of sustained static force in man. Neuroreport 7:2103-2110

Dupont AC, Morin EL (1994) A myoelectric control evaluation and trainer system. IEEE Trans Rehabil Eng 2:100-107

Evans HB, Pan Z, Parker PA, Scott RN (1984) Signal processing for proportional myoelectric control. IEEE Trans Biomed Eng 31:207-211

Gaine WJ, Smart C, Bransby-Zachary M (1997) Upper limb traumatic amputees. Review of prosthetic use. J Hand Surg [Br] 22:73-76

Gaines BR (1969) Linear and nonlinear models of human controller. Int J Man-Machine Studies 1:333-360

Gallant PJ, Morin EL, Peppard LE (1998) Feature-based classification of myoelectric signals using artificial neural networks. Med Biol Eng Comput 36:485-489

Gandevia SC, Kilbreath SL (1990) Accuracy of weight estimation for weights lifted by proximal and distal muscles of the human upper limb. Journal of Physiology (London) 423:299-310

Gandevia SC, McCloskey DI, Burke D (1992) Kinaesthetic signals and muscle contraction. Trends Neurosci 15:62-65

Georgopoulos AP, Pellizzer G, Poliakov AV, Schieber MH (1999) Neural coding of finger and wrist movements. J Comput Neurosci 6:279-288

Goodwin GM, McCloskey DI, Matthews PB (1972) Proprioceptive illusions induced by muscle vibration: contribution by muscle spindles to perception? Science 175:1382-1384

Gottlieb GL (1996) Muscle compliance: implications for the control of movement. Exerc Sport Sci Rev 24:1-34

Gregory JE, Proske U (1979) The responses of Golgi tendon organs to stimulation of different combinations of motor units. J Physiol 295:251-262

Hall LA, McCloskey DI (1983) Detections of movements imposed on finger, elbow and shoulder joints. J Physiol 335:519-533

Hogan N (1976) A review of the methods of processing EMG for use as a proportional control signal. Biomed Eng 11:81-86

Hogan N (1985) The mechanics of multi-joint posture and movement control. Biol Cybern 52:315-331

Hudgins B, Parker P, Scott RN (1993) A new strategy for multifunction myoelectric control. IEEE Trans Biomed Eng 40:82-94

Jami L (1992) Golgi tendon organs in mammalian skeletal muscle: functional properties and central actions. Physiol Rev 72:623666

Jones KE, Wessberg J, Vallbo AB (2001) Directional tuning of human forearm muscle afferents during voluntary wrist movements. J Physiol 536:635-647 
Jones LA (2000) Visual and haptic feedback in the control of force. Exp Brain Res 130:269-272

Kearney RE, Stein RB, Parameswaran L (1997) Identification of intrinsic and reflex contributions to human ankle stiffness dynamics. IEEE Trans Biomed Eng 44:493-504

Kirsch RF, Stein RB (1997) Neural and muscular properties: current views and controversies. In: Winters JM, Crago PE (eds) Biomechanics and neural control of movement. Springer, New York Berlin Heidelberg

Lovely DF, Stocker D, Scott RN (1990) A computer-aided myoelectric training system for young upper limb amputees. J Microcomput Appl 13:245-259

Lundberg A, Malmgren K, Schomburg ED (1978) Role of joint afferents in motor control exemplified by effects on reflex pathways from Ib afferents. J Physiol 284:327-343

Macefield G, Gandevia SC, Burke D (1990) Perceptual responses to microstimulation of single afferents innervating joints, muscles and skin of the human hand. J Physiol 429:113-129

Manal K, Gonzalez RV, Lloyd DG, Buchanan TS (2002) A real-time EMG-driven virtual arm. Comput Biol Med 32:25-36

Millstein SG, Heger H, Hunter GA (1986) Prosthetic use in adult upper limb amputees: a comparison of the body powered and electrically powered prostheses. Prosthet Orthot Int 10:27-34

Mirbagheri MM, Barbeau H, Kearney RE (2000) Intrinsic and reflex contributions to human ankle stiffness: variation with activation level and position. Exp Brain Res 135:423-436

Moberg E (1983) The role of cutaneous afferents in position sense, kinaesthesia, and motor function of the hand. Brain 106:1-19

Moran DW, Schwartz AB (1999) Motor cortical representation of speed and direction during reaching. J Neurophysiol 82:26762692

Morin E, Parker PA, Scott RN (1993) Operator error in a level coded myoelectric control channel. IEEE Trans Biomed Eng 40:558562

Mussa-Ivaldi FA, Hogan N, Bizzi E (1985) Neural, mechanical, and geometric factors subserving arm posture in humans. J Neurosci 5:2732-2743

Ng JK-F, Richardson CA, Kippers V, Parnianpour M, Bui BH (1996) Clinical applications of power spectral analysis of electromyographic investigations in muscle function. Man Ther 2:99-103
Nirkko AC, Ozdoba C, Redmond SM, Burki M, Schroth G, Hess CW, Wiesendanger M (2001) Different ipsilateral representations for distal and proximal movements in the sensorimotor cortex: activation and deactivation patterns. Neuroimage $13: 825-835$

Paciga JE, Richard PD, Scott RN (1980) Error rate in five-state myoelectric control systems. Med Biol Eng Comput 18:287290

Prochazka A, Wand P (1980) Tendon organ discharge during voluntary movements in cats. J Physiol 303:385-390

Rao AK, Gordon AM (2001) Contribution of tactile information to accuracy in pointing movements. Exp Brain Res 138:438-445

Reina GA, Moran DW, Schwartz AB (2001) On the relationship between joint angular velocity and motor cortical discharge during reaching. J Neurophysiol 85:2576-2589

Roeschlein RA, Domholdt E (1989) Factors related to successful upper extremity prosthetic use. Prosthet Orthot Int 13:14-18

Schwartz AB, Moran DW (2000) Arm trajectory and representation of movement processing in motor cortical activity. Eur J Neurosci 12:1851-1856

Scott RN (1966) Myoelectric control of prostheses. Arch Phys Med Rehabil 47:174-181

Scott RN, Parker PA (1988) Myoelectric prostheses: state of the art. J Med Eng Technol 12:143-151

Scott RN, Paciga JE, Parker PA (1978) Operator error in multistate myoelectric control systems. Med Biol Eng Comput 16:296301

Sears HH, Shaperman J (1991) Proportional myoelectric hand control: an evaluation. Am J Phys Med Rehabil 70:20-28

Stein RB, Zehr EP, Lebiedowska MK, Popovic DB, Scheiner A, Chizeck HJ (1996) Estimating mechanical parameters of leg segments in individuals with and without physical disabilities. IEEE Trans Rehabil Eng 4:201-211

Taira M, Boline J, Smyrnis N, Georgopoulos AP, Ashe J (1996) On the relations between single cell activity in the motor cortex and the direction and magnitude of three-dimensional static isometric force. Exp Brain Res 109:367-376

Thickbroom GW, Phillips BA, Morris I, Byrnes ML, Mastaglia FL (1998) Isometric force-related activity in sensorimotor cortex measured with functional MRI. Exp Brain Res 121:59-64

Viitasalo JH, Komi PV (1977) Signal characteristics of EMG during fatigue. Eur J Appl Physiol Occup Physiol 37:111-121

Zecca M, Micera S, Carrozza MC, Dario P (2002) Control of multifunctional prosthetic hands by processing the electromyographic signal. Crit Rev Biomed Eng 30:459-485 